\title{
Detection of outliers in the response and explanatory variables of the simple circular regression model
}

\begin{abstract}
The circular regression model may contain one or more data points which appear to be peculiar or inconsistent with the main part of the model. This may be occur due to recording errors, sudden short events, sampling under abnormal conditions etc. The existence of these data points ñoutliersò in the data set cause lot of problems in the research results and the conclusions. Therefore, we should identify them before applying statistical analysis. In this article, we aim to propose a statistic to identify outliers in the both of the response and explanatory variables of the simple circular regression model. Our proposed statistic is robust circular distance RCDxy and it is justified by the three robust measurements such as proportion of detection outliers, masking and swamping rates.
\end{abstract}

Keyword: Circular data; Circular regression; Outlier; Masking and swamping 